\title{
Value of the Multibiomarker Disease Activity Score to Predict Remission in RA: What Does the Evidence Show?
}

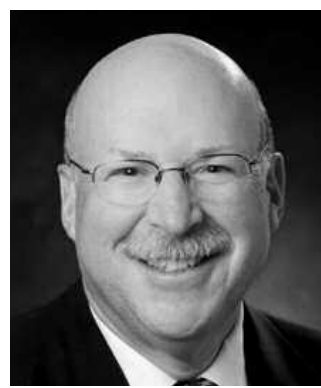

The achievement of true full clinical remission of rheumatoid arthritis (RA) is the goal of both patients and rheumatologists. There still is much discrepancy, with resulting confusion, in how remission is defined despite the consensus reached by the American College of Rheumatology and the European League Against Rheumatism that the most reliable definition of true remission is either a Boolean definition or a Simplified Disease Activity Index (SDAI) score $<3.3^{1}$. Other proposed remission cutoffs, including those for Disease Activity Score (DAS), 28-joint DAS (DAS28), and Routine Assessment of Patient Index Data 3 (RAPID-3), are much less stringent, and thus allow far more patients to be considered as having achieved "remission" despite the possibility of continued active disease in the joints with systemic inflammation (Figure 1) ${ }^{2}$. The accurate measurement of disease activity with a fully validated instrument is a key component of RA management.

Over the past decade there has been much interest in the discovery of biomarkers that can identify the complex and heterogeneous biology of RA and have the ability to reliably demonstrate the absolute degree of disease activity, both peripherally and systemically, in all patients. One instrument, which has claimed to be able to assess RA disease activity, predict radiographic progression, and achievement of remission, is the multibiomarker disease activity test (MBDA). Initially, 130 candidate serum protein biomarkers were selected from extensive literature screens, bioinformatics databases, mRNA expression, and protein microarray data. Of them, 25 were chosen and used to train an algorithm to purportedly measure disease activity, assessed by correlation to DAS28-C-reactive protein (CRP) and area under the receiver-operating characteristic curve for classification of low versus moderate/high disease activity. The final MBDA algorithm used 12 biomarkers: serum amyloid A, interleukin 6, tumor necrosis factor (TNF)-receptor 1, vascular endothelial growth factor-A, matrix metalloprotease (MMP)-1, chitinase-3-like-1 protein, MMP-3, epidermal growth factor, vascular cell adhesion molecule-1, leptin, resistin, and CRP. They were selected to mimic the components of the DAS28-CRP, including surrogates for tender and swollen joints, patient's global assessment of RA, and the CRP, to generate an MBDA score between 1 and $100^{3}$. The algorithm was then studied in diverse RA cohorts and "correlated" to RA clinical disease activity with resulting definitions of low $(\leq 29)$, moderate $(>29$ to $<44)$, and severe disease activity $(\geq 44)^{4}$. Unfortunately, the weakest metric among our tools was chosen to measure states of disease activity, the DAS28-CRP, which has been shown to overestimate control of disease activity much more frequently than the DAS, DAS28-erythrocyte sedimentation rate (ESR), SDAI, and the Clinical Disease Activity Index $(\mathrm{CDAI})^{5}$.

The ability of the MBDA to assess disease activity and predict radiographic progression or remission has not been assessed in prospective, randomized, controlled studies properly designed to answer a specific question. There are multiple reports of posthoc, retrospective analyses performed in patient cohorts that have concluded that the MBDA does have the ability to assess disease activity and predict these endpoints. For example, a posthoc analysis of the OPERA study found that the baseline MBDA score was independently associated with radiographic progression at 1 year and changes in MBDA score from baseline to 3 months with clinical remission, defined as DAS28-CRP $<2.6$, at 6 months. In patients who were positive for anticyclic citrullinated peptide antibodies (anti-CCP), 39\% with high MBDA score had radiographic progression versus 0 of 15 patients with low/moderate MBDA score. The authors concluded that early changes in MBDA score were associated with clinical remission based on DAS28-CRP at 6 months and that in anti-CCP-positive patients, a non-high baseline MBDA score had clinical value by predicting very low risk of radiographic progression at 12 months ${ }^{6}$.

Another example is a posthoc analysis of the POET study.

See MBDA predicting remission in RA, page 460

Personal non-commercial use only. The Journal of Rheumatology Copyright (C) 2019. All rights reserved. 


\section{SC abatacept plus MTX}

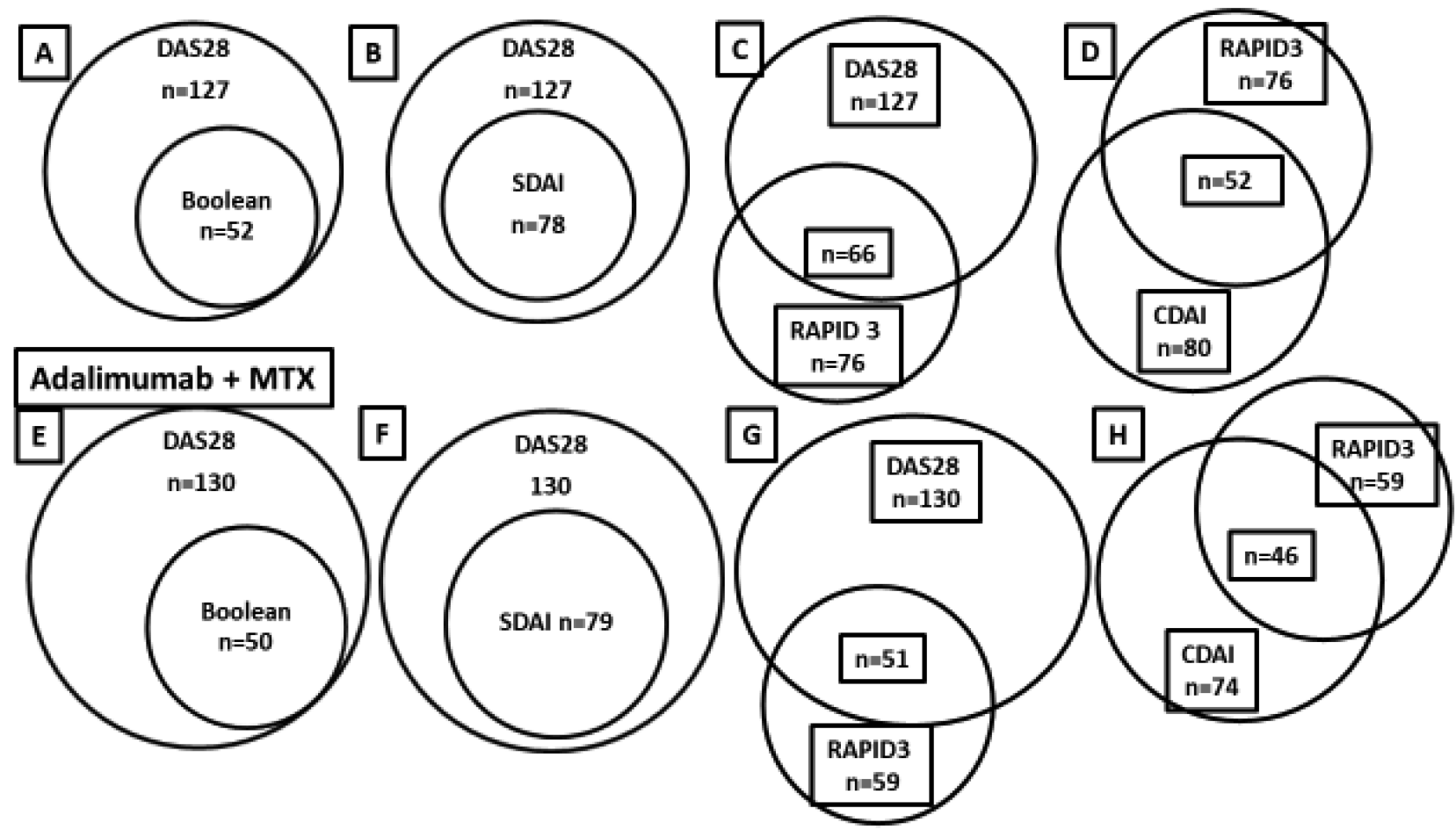

Figure 1. Relationship between disease measures defining remission in the AMPLE trial (Abatacept Versus Adalimumab Comparison in Biologic-Naive RA Subjects with Background Methotrexate). Each of the diagrams shows the percentage of patients achieving either DAS28-CRP < 2.6 ("remission") or RAPID-3 "remission," and of those, the percentage of patients who achieved Boolean, SDAI, or CDAI remission. Panels A-D: SC abatacept + MTX arm. Panels E-H: SC adalimumab + MTX. RA: rheumatoid arthritis; DAS28-CRP: 28-joint Disease Activity Score using C-reactive protein; RAPID-3: Routine Assessment of Patient Index Data 3; Boolean: Boolean remission; SDAI: Simple Disease Activity Index; CDAI: Clinical DAI; SC: subcutaneous; MTX: methotrexate.

Patients with stable low disease activity (LDA) who stopped TNF inhibitors (TNFi), a high baseline MBDA score was independently predictive of disease relapse within 12 months ${ }^{7}$.

In a posthoc analysis of a Dutch cohort of patients treated only with conventional synthetic disease-modifying antirheumatic drugs (DMARD), the MBDA score was associated with radiographic progression after adjustments for other risk factors. The MBDA score significantly differentiated risk for progression among seropositive patients when swollen joint count, CRP, or DAS28-CRP were low ${ }^{8}$.

A number of independent reports, however, have not corroborated these findings, and have suggested that the MBDA score is of little if any value in RA.

A posthoc analysis of the DRESS study evaluated the predictive value of the baseline MBDA score in longstanding RA patients with LDA who were tapering TNFi for successful tapering or discontinuation, occurrence of flare and major flare, and radiographic progression. The authors found that in this disease activity-guided strategy study, baseline MBDA score was not predictive for successful tapering, discontinuation, flare, major flare, or radiographic progression $^{9}$.
In a study to investigate predictors of flare in patients with RA who had LDA and to evaluate the effect of flare on 12-month clinical outcomes, it was found that at time of flare, although DAS28 and its components significantly correlated with MBDA, the correlation coefficients were low, and two-thirds of flares were not associated with a rise in biomarkers ${ }^{10}$.

A prespecified analysis from the AMPLE study assessed the adjusted mean change from baseline in the MBDA score. No association between the MBDA score and disease activity defined by the CDAI, SDAI, DAS28-CRP, or RAPID-3 in the abatacept and adalimumab treatment groups was observed. No correlation was found between disease activity assessed by the MBDA and radiographic progression ${ }^{11}$.

The RETRO study prospectively analyzed the risk for disease relapses in patients with RA in sustained remission (defined as a DAS28-ESR < 2.6) who were continuing, tapering, or stopping DMARD. Patients had documented RA with sustained DAS28-ESR $<2.6$ for $>6$ months. Relapse was defined as leaving DAS28 remission (DAS28-ESR $>2.6$ ). The authors found that the incidence of relapse was related to study arms regarding DMARD (continuing: $15.8 \%$; 
tapering: $38.9 \%$; stopping: $51.9 \%$; $\mathrm{p}=0.007)^{12}$. Relapses occurred particularly in the first 6 months after treatment reduction and were associated with the presence of anticitrullinated protein antibodies (ACPA).

A posthoc analysis of RETRO analyzed the ability of the MBDA to predict remission ${ }^{13}$. MBDA scores $>30$ were found in 33\% of patients, even though they were in remission. Of the patients who relapsed, 58\% had an MBDA $>30$ compared to $21 \%$ of patients who remained in remission. Multivariate regression analysis identified MBDA scores as an independent predictor for relapses in addition to ACPA status. Relapse rates were $13 \%$ in patients who were MBDA-/ACPA-, $33.3 \%$ in patients who were MBDA+/ ACPA-, $31.8 \%$ in patients MBDA-/ACPA+, and $76.4 \%$ in patients MBDA+/ACPA+. The authors concluded that the MBDA score significantly improved the prediction of relapses in patients with RA in stable remission undergoing DMARD tapering. However, one can view these results very differently; despite not being ACPA+ and having an MBDA $<30$, more than 1 in 8 patients still relapsed and neither the ACPA nor MBDA predicted this possibility. In addition, 1 in 4 patients, despite having a positive ACPA and MBDA $>30$, did not flare and could benefit from medication tapering or discontinuation, with a significant saving of medication cost. This raises the important question of whether a prudent rheumatologist or a knowledgeable and concerned patient would decide to stop or not stop a DMARD based on these odds. I would venture that a considerable number would not.

A major concern is that the results of these analyses favoring the use of the MBDA are being overinterpreted and overstated. The MBDA has not met the test of being a biomarker that can accurately and reliably predict who will or will not respond to a specific therapy, who will or will not be able to taper or stop therapy, or who will or will not have radiographic progression. Some would suggest that the MBDA is another tool to help in the decision-making process. But is the MBDA worth the expense (which is considerable) when the prudent physician and knowledgeable patient will still rely on a careful history, patient-reported symptoms, physical examination including the joints, markers of inflammation, and the judicious use of imaging, all of which are designed to provide metrics that can effectively be used in treatment management decisions? Many nonbiased analyses have demonstrated that the MBDA adds little to what we already know (and at a significant cost).

An article in this issue of The Journal by Hagen, et al, addresses the cost-effectiveness of being able to discontinue DMARD, particularly biological DMARD ${ }^{14}$. If a patient is in medication-free remission with complete control of their RA, their cost of treatment will be dramatically lower than that of a patient who does require medication to control their disease. In 2014, the total cost per treated patient across indications for multiple biologic disease modifying drugs were reported; the medication costs ranged from US $\$ 17,017$ to $\$ 41,888^{15}$. In 2018 , these costs are undoubtedly higher. For this reason, the conclusion of this publication cannot be disputed - less medication equates to less cost. Whether the MBDA adds to the ability to predict remission remains an open question that will need to be answered in a prospective, well-designed study. Until there is indisputable evidence that the MBDA is useful in these situations, there is little value in using it in clinical decision making.

\section{ROY FLEISCHMANN ${ }^{\circledR}$, MD,}

Clinical Professor of Medicine,

Metroplex Clinical Research Center,

University of Texas Southwestern Medical Center, Dallas, Texas, USA.

Address correspondence to Dr. R. Fleischmann, 8144 Walnut Hill Lane, Suite 810, Dallas, Texas 75231, USA.

E-mail: rfleischmann@arthdocs.com

\section{REFERENCES}

1. Felson DT, Smolen JS, Wells G, Zhang B, van Tuyl LH, Funovits J, et al. American College of Rheumatology/European League Against Rheumatism provisional definition of remission in rheumatoid arthritis for clinical trials. Arthritis Rheum 2011;63:573-86.

2. Fleischmann R, Schiff M, Weinblatt ME, Maldonado M, Massarotti E, Yazici Y. Two-year results from the Ample Trial: Low disease activity and association between remission and changes in physical function and radiographic outcomes with subcutaneous abatacept or adalimumab. Arthritis Rheum 2013;65 Suppl 10:S209.

3. Centola M, Cavet G, Shen Y, Ramanujan S, Knowlton N, Swan KA, et al. Development of a multi-biomarker disease activity test for rheumatoid arthritis. PLoS One 2013;8:e60635.

4. Curtis JR, van der Helm A, van Mil AH, Knevel R, Huizinga TW, Haney DJ, et al. Validation of a novel multibiomarker test to assess rheumatoid arthritis disease activity. Arthritis Care Res 2012;64:1794-803.

5. Fleischmann R, van der Heijde D, Koenig AS, Pedersen R, Szumski A, Marshall L, et al. How much does disease activity score in 28 joints ESR and CRP calculations underestimate disease activity compared with the simple disease activity index? Ann Rheum Dis 2015;74:1132-7.

6. Brahe CH, Østergaard M, Johansen JS, Defranoux N, Wang X, Bolce R. Predictive value of a multi-biomarker disease activity score for clinical remission and radiographic progression in patients with early rheumatoid arthritis: a post-hoc study of the OPERA trial. Scand J Rheumatol 2018 Jul 9 (E-pub ahead of print).

7. Ghiti-Moghadam M, Lamers-Karnebeek FBG, Vonkeman HE, Ten Klooster PM, Tekstra J, Schilder AM. Multi-biomarker disease activity score as a predictor of disease relapse in patients with rheumatoid arthritis stopping TNF inhibitor treatment. PLoS One 2018;13:e0192425.

8. Li W, Sasso EH, van der Helm-van Mil AH, Huizinga TW. Relationship of multi-biomarker disease activity score and other risk factors with radiographic progression in an observational study of patients with rheumatoid arthritis. Rheumatology 2016;55:357-66.

9. Bouman CAM, van der Maas A, van Herwaarden N, Sasso EH, van den Hoogen FHJ, den Broeder AA. A multi-biomarker score measuring disease activity in rheumatoid arthritis patients tapering adalimumab or etanercept: predictive value for clinical and radiographic outcomes. Rheumatology 2017;56:973-80.

10. Bechman K, Tweehuysen L, Garrood T, Scott DL, Cope AP, Galloway JB, et al. Flares in rheumatoid arthritis patients with low disease activity: predictability and association with worse clinical outcomes. J Rheumatol 2018 Sep 1 (E-pub ahead of print). 
11. Fleischmann R, Connolly SE, Maldonado MA, Schiff M. Brief report: estimating disease activity using multi-biomarker disease activity scores in rheumatoid arthritis patients treated with abatacept or adalimumab. Arthritis Rheumatol 2016;68:2083-9.

12. Haschka J, Englbrecht M, Hueber AJ, Manger B, Kleyer A, Reiser $\mathrm{M}$, et al. Relapse rates in patients with rheumatoid arthritis in stable remission tapering or stopping antirheumatic therapy: interim results from the prospective randomised controlled RETRO study. Ann Rheum Dis 2016;75:45-51.

13. Rech J, Hueber AJ, Finzel S, Englbrecht M, Haschka J, Manger B, et al. Prediction of disease relapses by multibiomarker disease activity and autoantibody status in patients with rheumatoid arthritis on tapering DMARD treatment. Ann Rheum Dis 2016;75:1637-44.
14. Hagen M, Englbrecht M, Haschka J, Reiser M, Kleyer A, Hueber A, et al. Cost-effective tapering algorithm in patients with rheumatoid arthritis - combination of multibiomarker disease activity score and autoantibody status. J Rheumatol 2019;46:460-6.

15. Wu N, Lee YC, Shah N, Harrison DJ. Cost of biologics per treated patient across immune-mediated inflammatory disease indications in a pharmacy benefit management setting: a retrospective cohort study. Clinical Ther 2014;36:1231-41.

J Rheumatol 2019;46:443-6; doi:10.3899/jrheum.181061 